\title{
Optimizing implementation of universal HIV treatment coverage for people living with HIV in Senegal
}

Project SOAR

Follow this and additional works at: https://knowledgecommons.popcouncil.org/departments_sbsr-hiv

Part of the Demography, Population, and Ecology Commons, Family, Life Course, and Society Commons, Gender and Sexuality Commons, and the International Public Health Commons How does access to this work benefit you? Let us know!

\section{Recommended Citation}

Project SOAR. 2018. "Optimizing implementation of universal HIV treatment coverage for people living with HIV in Senegal," Project SOAR Activity Brief. Washington, DC: Population Council. 


\section{Optimizing Implementation of Universal HIV Treatment Coverage for People Living with HIV in Senegal}

Senegal plans a rapid scale up of HIV treatment for all people living with HIV (PLHIV), regardless of CD4 count or viral suppression. However, limited data exist on how to achieve sustained viral suppression among PLHIV in real world contexts where significant barriers to effective antiretroviral therapy (ART) delivery, uptake, and adherence persist. Such information is critical to inform the rollout of Senegal's national test and treat strategy.

Project SOAR and partners are addressing these knowledge gaps by developing and testing a universal coverage ART intervention among PLHIV who are not virally suppressed. ${ }^{1}$ Specifically, the study aims to:

- Determine the feasibility, fidelity, and costeffectiveness of individual case management compared to standard of care (SOC) in achieving sustained viral suppression.

- Assess the acceptability of HIV self-testing by people at high risk of HIV infection and whether the promotion of self-testing increases the number of newly diagnosed PLHIV in clinic settings.

- Assess the acceptability of iris scanning as a strategy to enhance the measurement of follow-up and retention of PLHIV receiving ART.

Research Partners: Johns Hopkins University (JHU); Enda Santé; Division de Lutte Contre le SIDA, Ministry of Health $(\mathrm{MOH})$

Location: Dakar and Ziguinchor

Study Duration: 2015-2019

For more information, contact Stefan Baral, Johns Hopkins University, sbaral@jhu.edu.

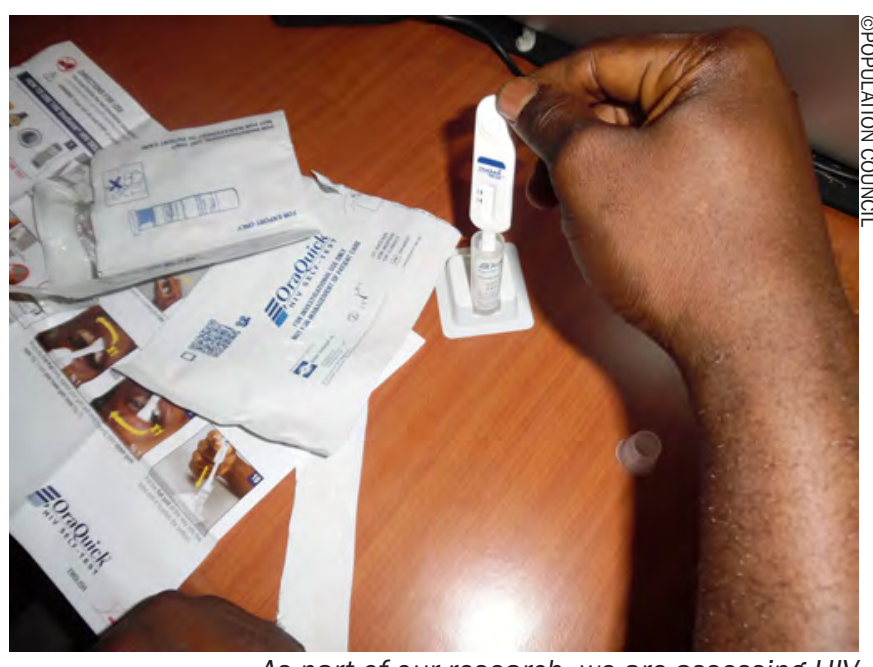

As part of our research, we are assessing HIV self-testing by people at high risk of HIV infection.

The results of this study will inform health care policy and practice in Senegal on the use of HIV self-testing approaches to increase the number of PLHIV diagnosed, case management interventions to improve care retention, and iris scanning to support measurement of retention in ART programs. Given Senegal's leadership role across West and Central Africa, the results are likely to inform implementation of test and treat programs across this broad and diverse region.

\section{OUR RESEARCH}

This study is an individual-level randomized controlled trial that examines the impact of individual case management, compared to SOC, on

${ }^{1}$ This study is part of a portfolio of three studies-one each in Senegal, Namibia and Nigeria-that SOAR and partners are conducting to evaluate strategies to improve treatment outcomes among PLHIV reached through test and treat approaches. For more information about these studies, visit our website: projsoar.org. 
viral suppression among PLHIV over a 12-month period. A total of 596 clients recruited from two government HIV treatment facilities in Ziguinchor and two in Dakar will be enrolled in the study. Study arms (SOC or case management) will be assigned to participants through individual randomization at the clinic level.

Data will be periodically collected from study participants using questionnaires and biological testing. In addition to viral suppression, other outcomes to be measured include mental health, social support, ART adherence, and stigma. For those who received a self-testing kit, the research team will inquire about use of the kit, test results, acceptability, and feasibility. To assess the costeffectiveness of case management, the researchers will determine the costs to clients in accessing and maintaining participation in the intervention as well as the costs to the health system in providing case management services. We will analyze the data by sex as well as gender identification.

\section{How will the HIV self-testing kits be used?}

Trained peers will distribute the HIV self-testing kits to high-risk and hard-to-reach populations, including men who have sex with men, female sex workers, and transgender women, to facilitate recruitment into the study. The trained peers will provide pre-test counseling on proper kit use, as well as the importance of confirmatory testing, irrespective of an HIV positive result. They will emphasize that the kit is a screening test and not meant to be used as a replacement for HIV testing at a health facility. For those who test positive with the HIV self-testing kits, there will be a referral to a study facility in order to facilitate linkage to care and treatment.

\section{What is case management?}

Case management is a multi-step process to coordinate care and provide a family-like support system for PLHIV. It involves face-to-face meetings between the case manager and the client, automatic text message sent to the client, and phone calls from the case manager. Case management approaches help address barriers to treatment uptake, adherence, and retention by working with individuals to set treatment goals, build treatment literacy and self-efficacy to execute treatment plans, and provide social and logistics support to patients to achieve their goals.

\section{Research Utilization}

We will employ a comprehensive research utilization strategy to engage stakeholders throughout the study design, data collection, results interpretation, and findings dissemination processes. Engagement with stakeholders aims to ensure local and international support of the study, alignment of the study with country data needs and priorities, and eventual translation of findings into programs and policies.

Our study team consists of JHU, Enda Santé, and Senegal $\mathrm{MOH}$ investigators to ensure engagement, ownership, and utilization of results by all parties. We have developed specific indicators for this study that will be used to track results uptake. These include assessing whether:

- Results are used to guide service delivery in Senegal,

- National policies are changed as noted in the Senegalese Country Operational Plan,

- Stakeholders are engaged throughout the study phases,

- Study aligns with country priorities, and

- There is community representation in a study oversight committee. agreement funded by the President's Emergency Plan for AIDS Relief and the U. S. Agency for International Development (Agreement No. AIDOAA-A-14-00060). SOAR is able to accept funding from all USAID accounts.
Project SOAR/Population Council

4301 Connecticut Avenue, NW, Suite 280

Washington, DC 20008

Tel: +1202 2379400

e-mail: ProjectSOAR@popcouncil.org

projsoar.org

()Population Council, March 2018 